\title{
Equipment Support Grid Architectures Based on WSRF
}

\author{
Wenjun $\mathrm{Li}^{*}$ and Xueqiang Yang \\ Department of Technical Support Engineering, Academy of Force Engineering, Beijing 100072, China \\ *Corresponding author
}

\begin{abstract}
Grid technology as a new information technology enables resource sharing and the "two fog" eliminating in the field of equipment support. Firstly, the demands of equipment support grid design were analyzed. Besides, the three popular grid architectures were outlined. Finally, the equipment support grid architectures were designed combined with the WSRF specifications in this paper to provide reference for the equipment support informatization construction.
\end{abstract}

Keywords—equipment support; grid; architectures; WSRF

\section{INTRODUCTION}

There exits "two fog" in the field of equipment support restricting its construction. One fog is the support demand fog problem. The species, quantities and units of equipment support demand are unclear. The other one is the support resource fog problem. The location, species, quantities and flows of equipment support resource are unknown. As a result of this, large scale, serious wasting, slow reaction speed and low efficiency exit in current equipment support systems. In order to improve the equipment support management level, a series of management information system achieving the office automation have been developed. However, the "two fog" problem remains unsolved. Every operation system keeps selfcontained just like the chimney. A new generation of information technology has spawned the development of equipment support management systems. Grid technology as a new information technology enables resource sharing and the "two fog" eliminating [1-3]. It has the characteristics of distribution, sharing, self-similarity, dynamics, diversity, autonomy and management multiplicity [4] which have a high consistency with the equipment support systems. Therefore, exploration on equipment support grid and equipment support grid architectures design are made in this paper.

\section{DEMANDS ANALYSIS}

\section{A. Military Demands Analysis}

The military demands of equipment support grid design are organizing all kinds of armored equipment support resources effectively in different regions, departments and organizations and providing efficient and reliable equipment support grid services for the army user according to the task, for example equipment support resource organization and management, data information interaction, data management, etc..

\section{B. Function Demand Analysis}

The equipment support grid systems need to provide the environment where every kind of operation systems and other equipment support resources can be integrated and shared and multi-hierarchy cooperation can be realized. The grid systems need to support the virtualization of the equipment support resources and encapsulate it into the form of web services for computer expression, network access and effective management of resources. What's more, the grid systems should be able to support the organization and implementation of the entire equipment support businesses and make reasonable combinations of web services in the grid systems according to the demands of task including equipment support business processes modeling, service retrieval, selection, binding and scheduling, monitoring of the implementation of business processes, etc..

\section{GRID ARCHITECTURES}

At present, grid technology has three popular architectures. One is the Five Layers Sandglass Architecture (FLSA) [5] proposed by Ian Foster in 2001.According to the distance between the components of the structure and the shared resources, functions of resources operation, management, and usage in the structure are distributed in five different layers [6]. Layers from the bottom up are the construction layer, the connection layer, the resource layer, the convergence layer and the application layer. The more the upper layer, the higher the degrees of resources sharing and the lower the degrees of individuality are. FLSA is essentially a stratification of protocols, and the numbers of protocols in every layer are different. The core protocol layers are the resource layer and the connection layer. It is protocol-centric and emphasizes the importance of services, application programming interfaces and software development kits, focusing on qualitative descriptions of protocols rather than specific definitions of protocols.

The other one is the Open Gird Services Architecture (OGSA) [7-8]. Based on FLSA and combined with web service technology, it was proposed. In the OGSA, all the shared resources in the grid system are abstracted into services applying mature web service technology and the basic services can be combined with task demands met. Every layer defines clearly corresponding functions in the OGSA model. The core layers are OGSA core service layer and Open Grid Service Infrastructure (OGSI) core service layer. OGSA is servicecentric. The concept of Grid Service is introduced in OGSI 
solving the two standard service interface definition and protocol identification problems.

Practice has proved that several problems exit in OGSI. For example, the normative contents are complicated and lack versatility and there are poor compatibility with Web Service tools and no distinction between service and status. In order to solve the contradiction between OGSI and the Web, Globus Alliance and IBM released the Web Service Resource Framework (WSRF) [9-10]. The WSRF specification eliminates the need for XML tools by transforming the original grid services into Web services and resource documents by transforming the functions of OGSI. WSRF is completely Web-based, and can be fully compatible with existing Web services tools and specifications. It achieves access to the status resources and has a good development prospects.

Therefore, equipment support grid architectures in this paper are designed based on WSRF specification and OGSA hierarchical grid design idea.

\section{EQUIPMENT SUPPORT GRID ARCHITECTURES}

According to the function demands, the equipment support grid architectures are divided into four different levels of resource layer, grid middleware layer, application layer and user interface layer, as shown in Figure1.

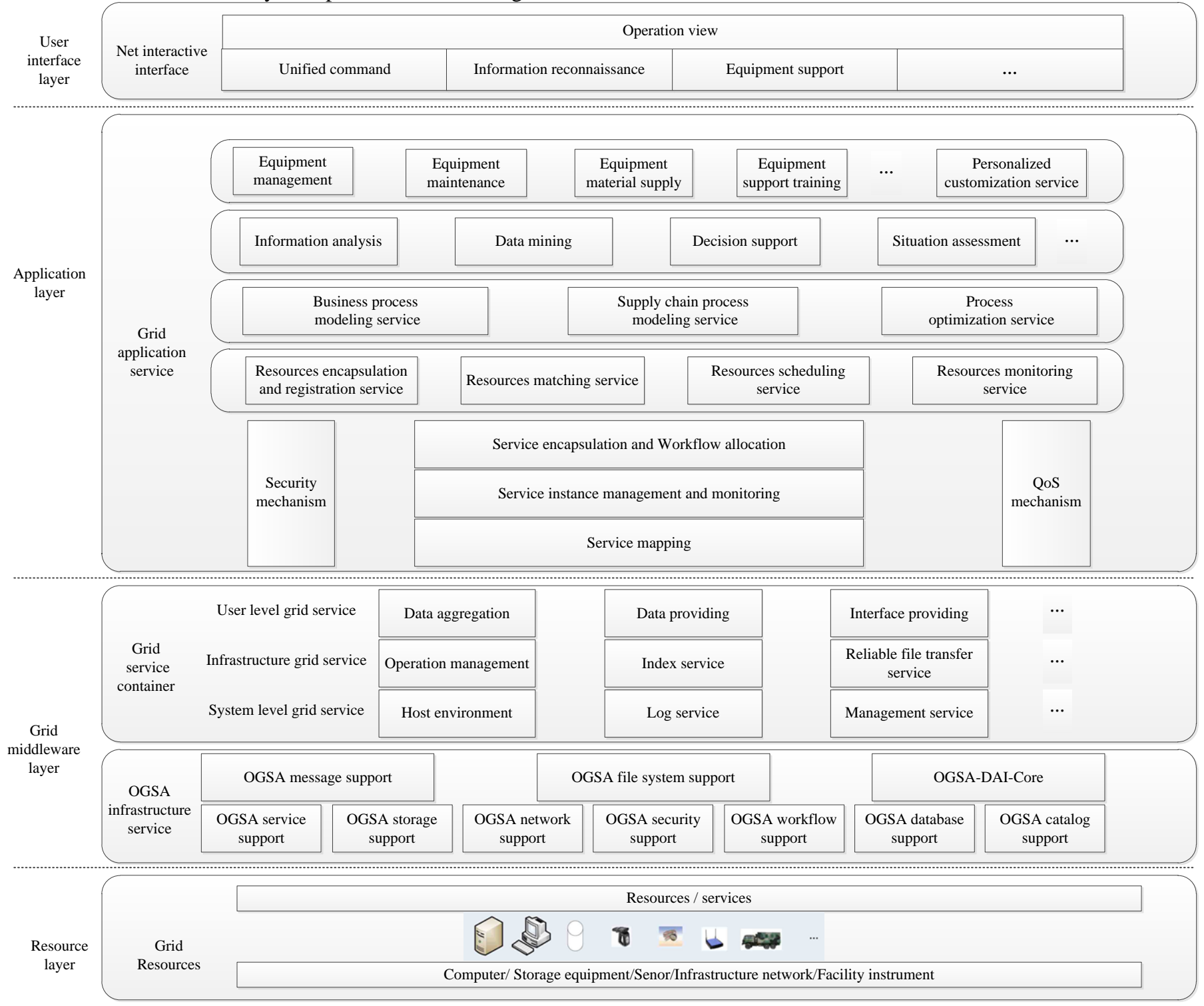

FIGURE I. EQUIPMENT SUPPORT GRID ARCHITECTURES BASED ON WSRF

1) Resource Layer: Resource layer is the architectural level of grid architectures at the bottom. Grid resources are the foundation of the grid providing every kind of service to users and satisfing equipment support task demands, which include resources involved in the equipment support process, for example, computer, storage resources, sensors, distributed systems, infrastructure network, equipment support facilities and so on.This layer provides shared resource capabilities and 
interfaces to the upper layer through grid services, enabling resources registration and access by supporting shared protocols.

2) Grid Middleware Layer: The grid infrastructure platform consists of two parts. One is the OGSA infrastructure service. The other is the grid universal service. It provides middleware services and program library for grid construction and grid applications. Shielding the distribution and heterogeneity of resources in the resources layer ae well as providing transparent and consistent using interface for the grid application layer and basic support environment for information discovery, resource management, data management, communication, security management, notification, monitoring and information services are its founction.

3) Application Layer: Application layer developes several dedicated services and tools for applications in equipment support. All levels provide services in the form of web services. Based on solving the problem of sharing and cooperative scheduling of various resource services, the encapsulation, registration, matching, scheduling, scheduling and monitoring of resources are realized through the encapsulation of service instances, the allocation of workflows, and the management and monitoring of service instances. Business process modeling services are completed by parsing specific business processes, calling process template libraries and business process constructors. Then, the supply chain process modeling services are completed by analyzing the supply process and calling the supply process template library and the business process constructor. Besides, process optimization services are proposed by analyzing process and providing process rationality detections. According to the specific demands of equipment support tasks, for example, equipment management, equipment maintenance, equipment material supply, equipment support training, and so on, finegrained services of the lower layer are combined and the corresponding business services are constructed to ensure the completion of equipment support tasks.

4) User Interface Layer: The user interface layer is the portal for the user accessing to the grid services, which can be flexibly configured according to the tasks requirements. After obtaining permission to access the eligibility, the user can access the grid through the browser. For ease of use, the interface should be simple and friendly.

\section{CONCLUSIONS}

Equipment support emphasizes time benefit of the task completion and maximizing military efficiency. Information highly sharing and accurate and fast delivery are the key elements in achieving this goal. Grid technology has such features. Based on WSRF specification and OGSA hierarchical grid design idea, equipment support grid architectures are designed in this paper providing a solution for the equipment support information construction. In the future work, the key technologies achieving the equipment support grid, equipment support resources management adapting to the grid dynamic and equipment support tasks coordination will be studied.

\section{REFERENCES}

[1] I. Foster, C. Kesselman, The Grid2: Blueprint for a new computing infrastructure, San Francisco: Morgan Kaufmann Publishers, 2004.

[2] I. Foster, C. Kesselman, and S.Tnecke, "The Anatomy of the Grid Enabling Scalable Virtual Organizations," International Journal of High Performance Computing Applications, Vo1.15, pp. 200-222, 2001.

[3] I. Foster, "The Grid: A New Infrastructure for 21st Century Science," Physics today, vol. 55, pp. 4247, 2002.

[4] X. Y. Qi, "Grid Technique and its Architecture," Science and Technology Innovation Herald, No. 33, pp. 16-18, 2009.

[5] X. L. Yang, Y. D. Wang, P. Xu, "A Survey of Developrnent of grid Architecture," Computer and Modernization, No. 8, pp. 33-36, 2010.

[6] J. L. Sun, "The Grid Technique," Office Informatization, No. 17, pp. 3334, 2016.

[7] J. Xu, Q. B. Liu, Y. S. Li, Service-oriented Grid Computing: A New Distributed Computing Architecture and Middleware, Beijing: .Science Press, 2009.

[8] L. Shi, "Research of Optimization for the Regional Logistic Information Service Composition based on Grid Technology," Beijing Jiaotong University, 2015.

[9] K. Czajkowski, "From Open Grid Services Infrastructure to WS_Resourse Framework Refactoring and Evolution," http://www. globus. org/wsrf/ specs/ ogsi_to_wsrf_1. 0. pdf,2006- 6- 2.

[10] Y. S. Sun, Y. Y. Yu, R. R. Qiu, J. H. Zhang, "Research on Grid Service Building Based on GDT," Information Science, vol. 32, pp. 119-121, 2014. 